\title{
The effect of volumetric breast density on the risk of screen-detected and interval breast cancers: a cohort study
}

\author{
Johanna O. P. Wanders ${ }^{1}$, Katharina Holland ${ }^{2}$, Nico Karssemeijer ${ }^{2}$, Petra H. M. Peeters ${ }^{1,3}$, Wouter B. Veldhuis ${ }^{4}$,
} Ritse M. Mann ${ }^{2}$ and Carla H. van Gils ${ }^{1 *}$

\begin{abstract}
Background: In the light of the breast density legislation in the USA, it is important to know a woman's breast cancer risk, but particularly her risk of a tumor that is not detected through mammographic screening (interval cancer). Therefore, we examined the associations of automatically measured volumetric breast density with screen-detected and interval cancer risk, separately.
\end{abstract}

Methods: Volumetric breast measures were assessed automatically using Volpara version 1.5.0 (Matakina, New Zealand) for the first available digital mammography (DM) examination of 52,814 women (age 50 - 75 years) participating in the Dutch biennial breast cancer screening program between 2003 and 2011. Breast cancer information was obtained from the screening registration system and through linkage with the Netherlands Cancer Registry. We excluded all screen-detected breast cancers diagnosed as a result of the first digital screening examination. During a median follow-up period of 4.2 (IQR 2.0-6.2) years, 523 women were diagnosed with breast cancer of which 299 were screendetected and 224 were interval breast cancers. The associations between volumetric breast measures and breast cancer risk were determined using Cox proportional hazards analyses.

Results: Percentage dense volume was found to be positively associated with both interval and screen-detected breast cancers (hazard ratio (HR) 8.37 (95\% Cl 4.34-16.17) and HR 1.39 (95\% Cl 0.82-2.36), respectively, for Volpara density grade category (VDG) 4 compared to VDG1 ( $p$ for heterogeneity $<0.001)$ ). Dense volume (DV) was also found to be positively associated with both interval and screen-detected breast cancers (HR $4.92(95 \% \mathrm{Cl} 2.98-8.12)$ and $\mathrm{HR}$ 2.30 (95\% Cl 1.39-3.80), respectively, for VDG-like category $(C) 4$ compared to C1 ( $p$ for heterogeneity $=0.041)$ ). The association between percentage dense volume categories and interval breast cancer risk (HR 8.37) was not significantly stronger than the association between absolute dense volume categories and interval breast cancer risk (HR 4.92).

Conclusions: Our results suggest that both absolute dense volume and percentage dense volume are strong markers of breast cancer risk, but that they are even stronger markers for predicting the occurrence of tumors that are not detected during mammography breast cancer screening.

Keywords: Volumetric mammographic breast density, Mammography screening, Interval breast cancer risk

\footnotetext{
*Correspondence: C.vanGils@umcutrecht.nl

1 Julius Center for Health Sciences and Primary Care, University Medical

Center Utrecht, P.O. Box 855003508 GA Utrecht, The Netherlands

Full list of author information is available at the end of the article
} 


\section{Background}

The female breast consists of fibroglandular (dense) and fat (nondense) tissue. It is known that breast density, measured as either the percentage or absolute amount of fibroglandular tissue in the breast, is a strong breast cancer risk factor [1-3]. Besides this, high breast density is also known to hinder the detection of tumors during mammographic screening [4-6]. These findings have led to legislation in the USA that mandates disclosure of breast density information to women undergoing mammography screening. Depending on their breast density, women may opt for supplemental screening, for example by ultrasound or magnetic resonance imaging (MRI).

When breast density measures are used as a tool to stratify women for different screening strategies, it is important that these measures are highly reproducible as it is undesirable that the likelihood of a woman being classified as having dense breasts is dependent on which radiologist she goes to. Another prerequisite is that these measures can be obtained in the daily practice of highthroughput screening.

Breast density can be measured using several methods. The most widely used methods are visual estimation using Breast Imaging - Reporting and Data System (BIRADS) density categories and the semi-automatic areabased Cumulus method [7]. The former is mostly used by radiologists in clinical and screening practice. The latter is mostly used for research purposes. Both methods have been shown to be related to breast cancer risk. However, they also have some disadvantages. BI-RADS density classification has high inter-reader variability and Cumulus is very time-consuming. Hence, neither method is optimal for stratification in breast cancer screening [8-12].

Nowadays, digital mammography (DM) is widely used and several software packages are available to measure breast density fully automatically on digital mammograms. Most packages report volumetric breast density measures, which are user-independent and developed with the intention to give an accurate and reproducible estimation of the amount of fibroglandular tissue in the breast using physics-based modeling based on information in the header of the raw digital images [13, 14]. This is in contrast to the aforementioned methods (BI-RADS and Cumulus) that measure the projected area of dense tissue on a mammogram. Density measured with automatic volumetric methods has also been examined in relation to breast cancer risk and results were found to be comparable to results from studies where BI-RADS or Cumulus were used to quantify breast density. In general, percentage dense volume (PDV) seemed to be a stronger breast cancer risk predictor than dense volume (DV) [15-18].Absolute dense volume represents the amount of fibroglandular tissue in the breast and is hypothesized to be positively associated with breast cancer risk as this is considered to be the actual tissue where breast cancers develop [19, 20]. Percent dense volume is the proportion of fibroglandular (dense) tissue in the breast. This has sometimes been suggested to be a stronger risk factor than absolute dense volume, which would indicate that the ratio between the two tissues is important, or that nondense volume, which is part of the denominator of percent dense volume, is a protective factor for breast cancer risk. In a large meta-analysis, Pettersson et al. previously studied the associations between both absolute and percentage dense area and breast cancer risk. Their results suggest that percentage dense area is a stronger risk indicator than absolute dense area, but some heterogeneity between study results remained [3]. By using automatically assessed volumetric breast density measures instead of projected area measures we hope to gain more insight into these ambiguities and to find the best measure to be used in breast cancer risk prediction.

In light of discussions on the need for supplemental screening after a negative mammogram, measures that indicate the risk of a tumor that is not detected at mammography screening (interval cancer) may be even more relevant. BI-RADS density and other area-based density measures have been shown to be strongly related to the risk of interval cancer [4, 21-26]. The relationship between automatic volumetric density methods and the risk of screen-detected and interval cancers, respectively, has not yet been studied. In this study, we used a fully automatic method to obtain several volumetric density measures, and assess their relationship with breast cancer risk. We distinguished between the risk of tumors that are detected at mammography (screen-detected cancers) or in the interval between two screening rounds (interval cancers), in a large cohort with digital mammograms from the population-based Dutch breast cancer screening program.

\section{Methods \\ Study population}

The study population consists of 52,814 women aged between 50 and 75 years who had one or more digital mammographic examinations at the Preventicon screening unit in Utrecht, the Netherlands, between 2003 and 2011. The Preventicon screening unit is one screening unit of the Foundation of Population Screening Mid-West screening region, which is one of the five screening regions of the Dutch biennial breast cancer screening program. All regions follow the same screening protocol. The Dutch program involves mammography only. By participating in the Dutch screening program, women consent to their data being used for evaluation and improvement of the screening, unless they have indicated otherwise. 


\section{Data collection}

In this breast cancer screening cohort, for each participant we collected the first unprocessed DM examination that was taken at the Preventicon screening unit between 2003 and 2011. All mammograms were acquired using Lorad Selenia DM systems (Hologic, Danbury, Conn.). If this was the participant's first screening examination within the program, this examination included the two standard views, craniocaudal (CC) and mediolateral oblique (MLO). If this was not her first screening examination within the program, i.e. when she had undergone film screen mammography in earlier rounds, MLO was the routinely acquired view and the $C C$ view was taken only when indicated (e.g. high breast density, visible abnormality). A CC view was present in $57 \%$ of the subsequent screening examinations. Information about follow up was obtained from the screening registration system. Women were followed until breast cancer diagnosis (event), death or until 2 years after the last available mammogram, whichever came first. Information on breast cancer development was obtained from the screening registration system and through linkage with the Netherlands Cancer Registry. This also included information on the mode of detection: through screening mammography (screen-detected cancer) or through diagnosis in the 24 months screening interval after a negative screening mammogram (interval cancer). Interval cancers diagnosed more than 24 months after a negative screening mammogram were not included for analysis. Within the screening program, information on risk factors other than age is not collected.

\section{Volumetric mammographic density assessment}

Absolute dense volume (DV), percentage dense volume (PDV) and absolute nondense volume (NDV) were automatically assessed from unprocessed mammograms of the left and right breasts, using Volpara density (version 1.5.0, Matakina, Wellington, New Zealand). Although normally both MLO and CC views would be used for calculating volumetric density measures with Volpara, here we used the mean of only the left and right MLO views for the risk analyses, since this is the routinely acquired view and $\mathrm{CC}$ views were not available for all women. In this way we ensured that breast density was assessed in the exact same way in all participants. Volpara density grades (VDGs) were constructed based on this average PDV using the standard Volpara cutoff points from version 1.5.0 (VDG1: 0\% $\leq$ PDV $<4.5 \%$, VDG2: $4.5 \% \leq \mathrm{PDV}<7.5 \%$, VDG3: $7.5 \% \leq \mathrm{PDV}<15.5 \%$, VDG4: PDV $\geq 15.5 \%)$. The VDGs are designed to mimic the fourth edition of the BI-RADS breast density categories. We chose to use this edition, because it is most comparable to what has been used in previous papers.

\section{Statistical analysis}

Descriptive statistics of age and breast measures (DV, PDV, NDV and VDG) were determined. Continuous breast measures were transformed using the natural logarithm (ln) to obtain normal distributions. Pearson correlation coefficients for correlation between different breast measures and between breast measures and age were determined. We also assessed the correlation between breast measures on the MLO and CC views among women who had both views available, and on the MLO views of the left and right breasts.

We examined the associations between the different breast density measures and breast cancer risk by calculating hazard ratios (HR) and their 95\% confidence intervals (95\% CI) using Cox proportional hazards analyses for quartiles (quartile cutoffs were determined based on the distribution of the different density measures in the whole cohort at baseline) and continuous measures (per standard deviation (SD) increase of the lntransformed measure) of DV and PDV. We also determined associations between breast cancer risk and two additional breast density measures: (1) VDG categories (Volpara BI-RADS) and (2) a categorized DV variable in which the cutoff points were created as to obtain the same group sizes as with the VDG categories (VDG-like categories).

To minimize the number of breast cancer cases in the model that were diagnosed based on the same mammogram as was used for breast density assessment, we excluded all screen-detected breast cancer cases of women diagnosed on the basis of their first digital screening examination.

Cox proportional hazards analyses were used to examine the association between breast density and breast cancer risk. Age was used as the underlying time scale with entry and exit time defined as age at time of the first available DM and age at breast cancer diagnosis, death or censoring 2 years after the last DM before 1 January 2012. In this way, the confounding effect of age was taken into account. Two models were constructed. The first model only contained one of the density measures. The second model was adjusted for NDV in case the model included quartiles, categories or continuous measures of DV. We found that DV and NDV volume were positively correlated (Pearson correlation $=0.50$ ). By adjusting for NDV we attempted to determine the independent effect of DV on breast cancer risk, without the interference of NDV. Models containing a PDV measure (including VDG) were not adjusted for NDV, since NDV is already part of PDV $(\mathrm{PDV}=\mathrm{DV} /(\mathrm{DV}+$ NDV)). The proportional hazards assumption was evaluated by Schoenfeld residual plots and log-minuslog plots, and the assumption was not violated. To 
examine the presence of a linear trend in HRs over the quartiles and categories of breast measures, quartile and category variables were added to the models as continuous variables.

To examine the association between the different density measures and screen-detected or interval cancers and to test whether the association was statistically significantly different between density measures and screen-detected versus interval cancers, the method of Lunn and McNeil was used for competing risk analysis $[27,28]$. Cox proportional hazards analysis was used for this method, with time of follow up as the underlying time scale. Age at time of first available DM was added as a covariate in the models to take the confounding effect of age into account.

A bootstrap method was used to determine whether measures based on PDV or DV were stronger breast cancer risk factors. This was done for VDG versus VDGlike categories, quartiles of PDV versus quartiles of DV and continuous PDV versus continuous DV. The differences between coefficients for models containing a PDV or DV measure were determined within 2000 bootstrap samples. A difference of 0 indicates that there is no difference between the effect of DV and PDV on breast cancer risk. A 95\% CI that does not include 0 means that one measure is statistically significantly more strongly related to breast cancer risk than the other measure.

In addition, a sensitivity analysis was performed where we only used the contralateral MLO view for breast cancer cases and for non-breast cancer cases the MLO view of a random chosen side, with the same left and right distribution as that of the breast cancer cases.

For the main analysis both invasive and in situ breast cancers were used. In a sensitivity analysis, we only took invasive breast cancers into account for data analysis.

For all breast density measures, we compared the fourth category or quartile with the first. Nowadays, often the second category is used as the reference category, as BI-RADS breast density category 2 is the most prevalent category among women of breast cancer screening age. To be able to compare with other studies, we also present results using the second category or quartile of each breast density measure as reference category. Statistical analyses were performed using SPSS version 22 and $\mathrm{R}$ version 3.2.0.

\section{Results}

After excluding cases of screen-detected breast cancer, diagnosed on the basis of the first available digital screening examination, 523 breast cancers, including 299 screen-detected and 224 interval breast cancers were used for analyses. At the first digital screening mammogram the median age was 56 years (IQR 51-63) in women who did not develop breast cancer $(\mathrm{N}=$
52,291), 59 years (IQR 54-64) in women who were diagnosed with screen-detected breast cancer and 55 years (IQR 50-62) in women diagnosed with interval breast cancer during follow up. The median total number of screening rounds in which women participated (analog (if applicable) and digital together) was 5 (IQR 2-8), 7 (IQR 4-9) and 4 (IQR 2-7) in women who were not diagnosed with breast cancer, were diagnosed with a screen-detected breast cancer and were diagnosed with interval cancer, respectively. The median follow-up time after the first digital mammogram until breast cancer diagnosis (event), death or end of follow up, was 4.2 years (IQR 2.0-6.2), 3.8 years (IQR 2.1-4.3) and 2.3 (IQR 1.34.0 ), in women with no diagnosis of breast cancer, screen-detected breast cancer and interval breast cancer, respectively (Table 1).

PDV was strongly negatively correlated with NDV $(-0.70, p<0.01))$ and positively correlated with DV $(0.27$, $p<0.01)$. DV was positively correlated with NDV $(0.50$, $p<0.01)$. DV and PDV were both negatively correlated with age $(-0.16$ and -0.29 , respectively, $p<0.01)$, whereas NDV was positively correlated with age $(0.14, p<0.01)$. There was strong correlation between breast measures of the left and right breast and between CC and MLO views. Correlations between the MLO views of the left and right breast for DV, PDV and NDV was 0.86, 0.91 and 0.98 , respectively $(\mathrm{N}=52,410$ and $p<0.01$ for all three). Correlation between MLO and CC views of the right breast was $0.86,0.90$ and $0.97 \mathrm{DV}$, PDV and NDV, respectively ( $\mathrm{N}=38,997$ and $p<0.01$ for all three).

\section{Association between breast density measures and breast cancer risk (total)}

In Fig. 1 and Table 2 it is shown that all breast density measures (VDG, quartiles of PDV, continuous PDV, VDGlike categories, quartiles of DV and continuous DV) were positively associated with breast cancer risk, with HR 3.14 (95\% CI 2.17-4.55) for VDG category 4 compared to category 1 and HR 3.55 (95\% CI 2.49-5.05) for VDG-like category 4 compared to category 1, and HR 2.42 (95\% CI 1.83-3.20) for PDV and 2.60 (95\% CI 1.96-3.45) for DV quartile 4 compared to quartile 1 . The risk estimates of PDV and DV measures were not statistically significantly different from one another (Table 3).

The HRs for continuous measures of PDV and DV were 1.36 (95\% CI $1.25-1.48$ ) and 1.45 (95\% CI $1.32-$ $1.60)$, respectively, for one standard deviation increase in the ln-transformed continuous measure (not statistically significantly different from one another (Table 3 )).

\section{Association between breast density measures and screen- detected breast cancer risk}

Figure 2 and Table 4 show the association between different breast density measures and the risk of screen- 
Table 1 Characteristics of 52,814 women undergoing digital mammography (first time) between 2003 and 2011

\begin{tabular}{|c|c|c|c|c|c|c|}
\hline \multirow[b]{2}{*}{ Age (years) ${ }^{a}$, median (IQR) } & \multicolumn{2}{|c|}{$\begin{array}{l}\text { No breast cancer } \\
(N=52,291)\end{array}$} & \multicolumn{2}{|c|}{$\begin{array}{l}\text { Screen-detected cancer } \\
(N=299)\end{array}$} & \multicolumn{2}{|c|}{$\begin{array}{l}\text { Interval cancer } \\
(N=224)\end{array}$} \\
\hline & 56 & $(51 ; 63)$ & 59 & $(54 ; 64)$ & 55 & $(50 ; 62)$ \\
\hline Number of screening rounds participated in, median (IQR) & 5 & $(2 ; 8)$ & 7 & $(4 ; 9)$ & 4 & $(2 ; 7)$ \\
\hline Follow up (years) ${ }^{b}$, median (IQR) & 4.2 & $(2.0 ; 6.2)$ & 3.8 & $(2.1 ; 4.3)$ & 2.3 & $(1.3 ; 4.0)$ \\
\hline \multicolumn{7}{|l|}{ VDG categories, $n(\%)$} \\
\hline Category 1 & 10,458 & $(20.0)$ & 46 & $(15.4)$ & 12 & $(5.4)$ \\
\hline Category 2 & 21,276 & $(40.7)$ & 143 & $(47.8)$ & 72 & $(32.1)$ \\
\hline Category 3 & 15,856 & $(30.3)$ & 89 & $(29.8)$ & 100 & $(44.6)$ \\
\hline Category 4 & 4,701 & $(9.0)$ & 21 & $(7.0)$ & 40 & $(17.9)$ \\
\hline \multicolumn{7}{|l|}{ Continuous density measures, median (IQR) } \\
\hline Dense volume $\left(\mathrm{cm}^{3}\right)^{a}$ & 57.7 & $(42.8 ; 78.8)$ & 63.2 & $(49.0 ; 83.5)$ & 67.7 & $(48.9 ; 93.2)$ \\
\hline Percent dense volume (\%) ${ }^{\mathrm{a}}$ & 6.4 & $(4.8 ; 9.8)$ & 6.5 & $(5.0 ; 9.4)$ & 8.5 & $(6.2 ; 13.6)$ \\
\hline Nondense volume $\left(\mathrm{cm}^{3}\right)^{\mathrm{a}}$ & 805.0 & $(518.8 ; 1183.7)$ & 929.1 & $(585.6 ; 1298.7)$ & 720.3 & $(469.8 ; 1030.9)$ \\
\hline Total breast volume $\left(\mathrm{cm}^{3}\right)^{a}$ & 866.8 & $(573.9 ; 1256.8)$ & 994.0 & $(648.6 ; 1376.5)$ & 797.5 & $(531.8 ; 1105.8)$ \\
\hline
\end{tabular}

VDG Volpara density grade

${ }^{a}$ At first digital screening mammogram

${ }^{b}$ Women were followed until breast cancer diagnosis (event), till death or till 2 years after the last available mammogram, whichever came first

detected breast cancer. The measures based on DV (VDG-like categories, quartiles of DV and continuous DV) were positively associated with the risk of screendetected breast cancer. The associations between PDV measures (VDG, quartiles of PDV and continuous PDV) and screen-detected breast cancer risk were increased, but were not statistically significant or were borderline significant.

The HRs were 2.30 (95\% CI 1.39-3.80) and 1.39 (95\% CI 0.82-2.36) for VDG-like category and VDG category 4 compared to category 1 , respectively and were 2.24 (95\% CI 1.51-3.31) and 1.36 (95\% CI 0.97-1.94) for the

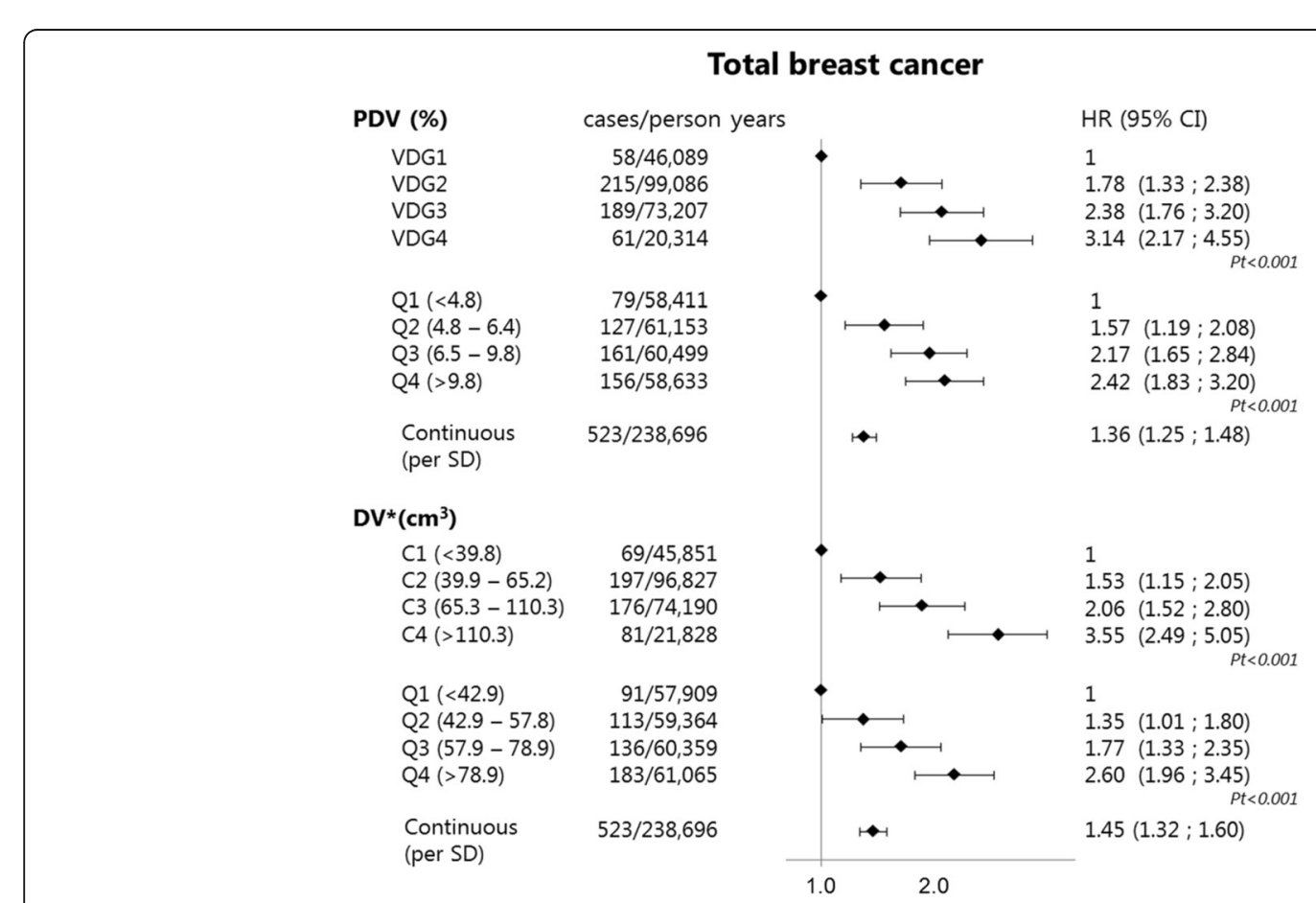

Fig. 1 Associations between mammographic measures and breast cancer risk. In the cox proportional hazards analyses age was used as the underlying time scale. Pt p-trend: this was determined by adding the categorical measures as a continuous measure to the model, PDV percentage dense volume, DV dense volume, Per SD per standard deviation, VDG Volpara density grade, Q quartile, C category. ${ }^{*}$ Absolute dense volume (DV) measures were adjusted for nondense (breast fat) volume 
Table 2 Association between mammographic measures and breast cancer risk

\begin{tabular}{|c|c|c|c|c|}
\hline & Cases/person years & $\mathrm{HR}_{1}(95 \% \mathrm{Cl})$ & $\mathrm{HR}_{2}(95 \% \mathrm{Cl})$ & $H R_{\text {invasive }}(95 \% \mathrm{Cl})$ \\
\hline \multicolumn{5}{|l|}{ Volpara density grades } \\
\hline VDG1 & $58 / 46,089$ & ref & NA & ref \\
\hline VDG2 & $215 / 99,086$ & $1.78(1.33 ; 2.38)$ & NA & $1.89(1.38 ; 2.58)$ \\
\hline VDG3 & $189 / 73,207$ & $2.38(1.76 ; 3.20)$ & NA & $2.53(1.83 ; 3.49)$ \\
\hline VDG4 & $61 / 20,314$ & $3.14(2.17 ; 4.55)$ & NA & $3.54(2.39 ; 5.24)$ \\
\hline$P$ trend & & $<0.001$ & & $<0.001$ \\
\hline \multicolumn{5}{|l|}{ Percent dense volume (in quartiles) } \\
\hline Q1 (<4.8\%) & $79 / 58,411$ & ref & NA & ref \\
\hline Q2 (4.8-6.4\%) & $127 / 61,153$ & $1.57(1.19 ; 2.08)$ & NA & $1.61(1.19 ; 2.18)$ \\
\hline Q3 (6.4-9.8\%) & $161 / 60,499$ & $2.17(1.65 ; 2.84)$ & NA & $2.33(1.74 ; 3.11)$ \\
\hline Q4 (>9.8\%) & $156 / 58,633$ & $2.42(1.83 ; 3.20)$ & NA & $2.52(1.87 ; 3.40)$ \\
\hline$P$ trend & & $<0.001$ & & $<0.001$ \\
\hline Percent dense volume (per SD increase) & $523 / 238,696$ & $1.36(1.25 ; 1.48)$ & NA & $1.38(1.26 ; 1.51)$ \\
\hline \multicolumn{5}{|l|}{ Dense volume (VDG-like categories) } \\
\hline $\mathrm{Cl}\left(<39.8 \mathrm{~cm}^{3}\right)$ & $69 / 45,851$ & ref & ref & ref \\
\hline $\mathrm{C} 2\left(39.9-65.2 \mathrm{~cm}^{3}\right)$ & $197 / 96,827$ & $1.37(1.04 ; 1.80)$ & $1.53(1.15 ; 2.05)$ & $1.69(1.24 ; 2.30)$ \\
\hline C3 $\left(65.3-110.1 \mathrm{~cm}^{3}\right)$ & $176 / 74,190$ & $1.66(1.26 ; 2.20)$ & $2.06(1.52 ; 2.80)$ & $2.27(1.63 ; 3.14)$ \\
\hline $\mathrm{C} 4\left(>110.1 \mathrm{~cm}^{3}\right)$ & $81 / 21,828$ & $2.79(2.02 ; 3.86)$ & $3.55(2.49 ; 5.05)$ & $3.66(2.50 ; 5.36)$ \\
\hline$P$ trend & & $<0.001$ & $<0.001$ & $<0.001$ \\
\hline \multicolumn{5}{|l|}{ Dense volume (in quartiles) } \\
\hline Q1 $\left(<42.9 \mathrm{~cm}^{3}\right)$ & $91 / 57,909$ & ref & ref & ref \\
\hline Q2 $\left(42.9-7.8 \mathrm{~cm}^{3}\right)$ & $113 / 59,364$ & $1.22(0.93 ; 1.61)$ & $1.35(1.01 ; 1.80)$ & $1.47(1.08 ; 1.99)$ \\
\hline Q3 $\left(57.9-78.9 \mathrm{~cm}^{3}\right)$ & $136 / 60,359$ & $1.48(1.13 ; 1.93)$ & $1.77(1.33 ; 2.35)$ & $1.92(1.42 ; 2.60)$ \\
\hline $\mathrm{Q} 4\left(>78.9 \mathrm{~cm}^{3}\right)$ & $183 / 61,065$ & $2.08(1.61 ; 2.68)$ & $2.60(1.96 ; 3.45)$ & $2.71(2.01 ; 3.67)$ \\
\hline$P$ trend & & $<0.001$ & $<0.001$ & $<0.001$ \\
\hline Dense volume (per SD increase) ${ }^{\#}$ & $523 / 238,696$ & $1.34(1.23 ; 1.46)$ & $1.45(1.32 ; 1.60)$ & $1.45(1.32 ; 1.60)$ \\
\hline
\end{tabular}

$H R_{1}$ Cox proportional hazards analysis where age was used as the underlying time scale, $H R_{2}$ dense volume HR's are adjusted for nondense volume (quartiles), $H R_{\text {invasive }}$ Cox proportional hazards analysis with only invasive breast cancers, where age was used as the underlying time scale. Dense volume HRs were adjusted for nondense volume (quartiles), NA Not applicable

$P$ trend was determined by adding the categorical measures as a continuous measure into the model

\#Continuous measures were natural-logarithm-transformed to establish normal distributions

highest compared to the lowest quartiles of DV and PDV, respectively. The HRs were 1.27 (95\% CI 1.14$1.43)$ and $1.10(0.97-1.25)$ for continuous measures of DV and PDV, respectively. DV, measured continuously, had a significantly stronger association with screendetected breast cancer risk than continuously measured PDV (Table 3). Although DV was significantly associated with screen-detected breast cancer risk, and PDV was not, the risk estimates for VDG versus VDG-like categories and PDV versus DV quartiles in relation to screen-detected breast cancer risk were not statistically significantly different (Table 3 ).

\section{Association between breast density measures and interval breast cancer risk}

The association between breast density measures and interval breast cancer risk is presented in Fig. 2 and
Table 5 . All breast density measures were statistically significantly associated with increased risks of interval breast cancer. The HRs were 4.92 (95\% CI 2.98-8.12) and 8.37 (95\% CI 4.34-16.17) for VDG-like category and VDG category 4 compared to category 1 , respectively and 2.79 (95\% CI 1.86-4.19) and 5.89 (95\% CI 3.42-10.14) for the highest compared to the lowest quartiles of DV and PDV, respectively. Quartiles of PDV were more strongly associated with interval breast cancer risk than quartiles of DV, and this difference was statistically significant (Table 3). The difference in effect between VDG versus VDG-likecategories was not statistically significantly different. The continuous measures of PDV and DV were also positively associated with interval breast cancer risk, with HRs of 1.65 (95\% CI 1.45-1.87) and 1.63 (95\% CI 1.42-1.87) for one standard deviation increase in the ln-transformed continuous measure (not significant (Table 3)). 
Table 3 Percentage dense volume versus dense volume measures in relation to breast cancer risk (bootstrap analysis results)

\begin{tabular}{|c|c|c|c|}
\hline Total breast cancers & $\begin{array}{l}\text { Significance of the difference between } \\
\text { coefficients in PDV and DV models }\end{array}$ & $\begin{array}{l}\text { Difference between coefficients } \\
\text { in PDV and DV models }\end{array}$ & $\begin{array}{l}95 \% \mathrm{Cl} \text { of difference between } \\
\text { coefficients in PDV and DV models }\end{array}$ \\
\hline \multirow[t]{3}{*}{ VDG vs VDG-like categories } & \multirow[t]{3}{*}{ Non significant } & C2: 0.15 & $(-0.25 ; 0.56)$ \\
\hline & & C3: 0.15 & $(-0.23 ; 0.52)$ \\
\hline & & C4: -0.11 & $(-0.51 ; 0.27)$ \\
\hline \multirow[t]{3}{*}{ Quartiles PDV vs DV } & \multirow[t]{3}{*}{ Non significant } & Q2: 0.16 & $(-0.24 ; 0.54)$ \\
\hline & & Q3: 0.21 & $(-0.17 ; 0.57)$ \\
\hline & & Q4: - 0.07 & $(-0.37 ; 0.24)$ \\
\hline Continuous PDV vs DV & Non significant & -0.07 & $(-0.15 ; 0.01)$ \\
\hline \multicolumn{4}{|l|}{ Screen-detected cancers } \\
\hline \multirow[t]{3}{*}{ VDG vs VDG-like categories } & \multirow[t]{3}{*}{ Non significant } & C2: -0.03 & $(-0.56 ; 0.46)$ \\
\hline & & C3: -0.17 & $(-0.64 ; 0.28)$ \\
\hline & & C4: -0.49 & $(-1.08 ; 0.07)$ \\
\hline \multirow[t]{3}{*}{ Quartiles PDV vs DV } & \multirow[t]{3}{*}{ Non significant } & Q2: -0.14 & $(-0.65 ; 0.32)$ \\
\hline & & Q3: -0.14 & $(-0.62 ; 0.34)$ \\
\hline & & Q3: -0.49 & $(-0.90 ;-0.12)$ \\
\hline Continuous PDV vs DV & Significant, stronger for DV & -0.13 & $(-0.24 ;-0.03)$ \\
\hline \multicolumn{4}{|l|}{ Interval cancers } \\
\hline \multirow[t]{3}{*}{ VDG vs VDG-like categories } & \multirow{3}{*}{$\begin{array}{l}\text { Non significant (except for C2 and C3: } \\
\text { stronger for PDV) }\end{array}$} & $C 2: 0.73$ & $(0.01 ; 1.56)$ \\
\hline & & C3: 0.92 & $(0.26 ; 1.68)$ \\
\hline & & C4: 0.58 & $(-0.06 ; 1.28)$ \\
\hline \multirow[t]{3}{*}{ Quartiles PDV vs DV } & \multirow[t]{3}{*}{ Significant, stronger for PDV } & Q2: 0.90 & $(0.21 ; 1.60)$ \\
\hline & & Q3: 1.02 & $(0.40 ; 1.66)$ \\
\hline & & Q3: 0.77 & $(0.26 ; 1.34)$ \\
\hline Continuous PDV vs DV & non significant & 0.01 & $(-0.11 ; 0.13)$ \\
\hline
\end{tabular}

VDG Volpara density grade, PDV percentage dense volume, DV dense volume

Significant: none of the bootstrap 95\% Cls for differences between quartile (Q)2, Q3, or Q4 (or category (C)2, C3, or C4) contain zero, otherwise they were non-significant

Bold text means that the difference between coefficients in PDV an DV models are significant

For all breast measures (except quartiles of DV ( $p=$ $0.355)$ ) the risk in relation to interval breast cancer was statistically significantly stronger than that in relation to screen-detected breast cancer (Fig. 2). The associations between percentage dense volume and interval breast cancer risk seemed somewhat stronger than the associations between absolute dense volume and interval breast cancer risk, although this difference between percentage and absolute dense volume was not consistently statistically significant (Table 3 ).

Of the 523 breast cancers in our study, 56 (11\%) were in situ, and 466 (89\%) were invasive breast cancers. For one case of breast cancer, information on invasiveness was missing. Of the interval cancers, 218 (97\%) were invasive breast cancers compared to $83 \%$ of screendetected cancers. Results restricted to invasive breast cancers are presented in Tables 2, 4 and 5. In general, the effects for invasive breast cancer were similar to those for invasive and in situ cancers combined.
Our results did not change when we only used the contralateral MLO view for breast cancer cases and the MLO view of a random chosen side for non-breast cancer cases (instead of the mean of the left and right view) for breast density measurements in relation to breast cancer risk (data not shown).

For reference and for comparison with other papers, Additional file 1: Figure S1 and Additional file 2: Figure S2 are presented with the second instead of the first breast density categories as the reference category.

\section{Discussion}

In this study we found that automatically assessed volumetric breast density measures are positively associated with breast cancer risk and in particular with the risk of interval cancer. The difference in effect on interval and screen-detected cancers appeared slightly more pronounced for the percentage dense volume measures than for absolute dense volume measures. The VDG 


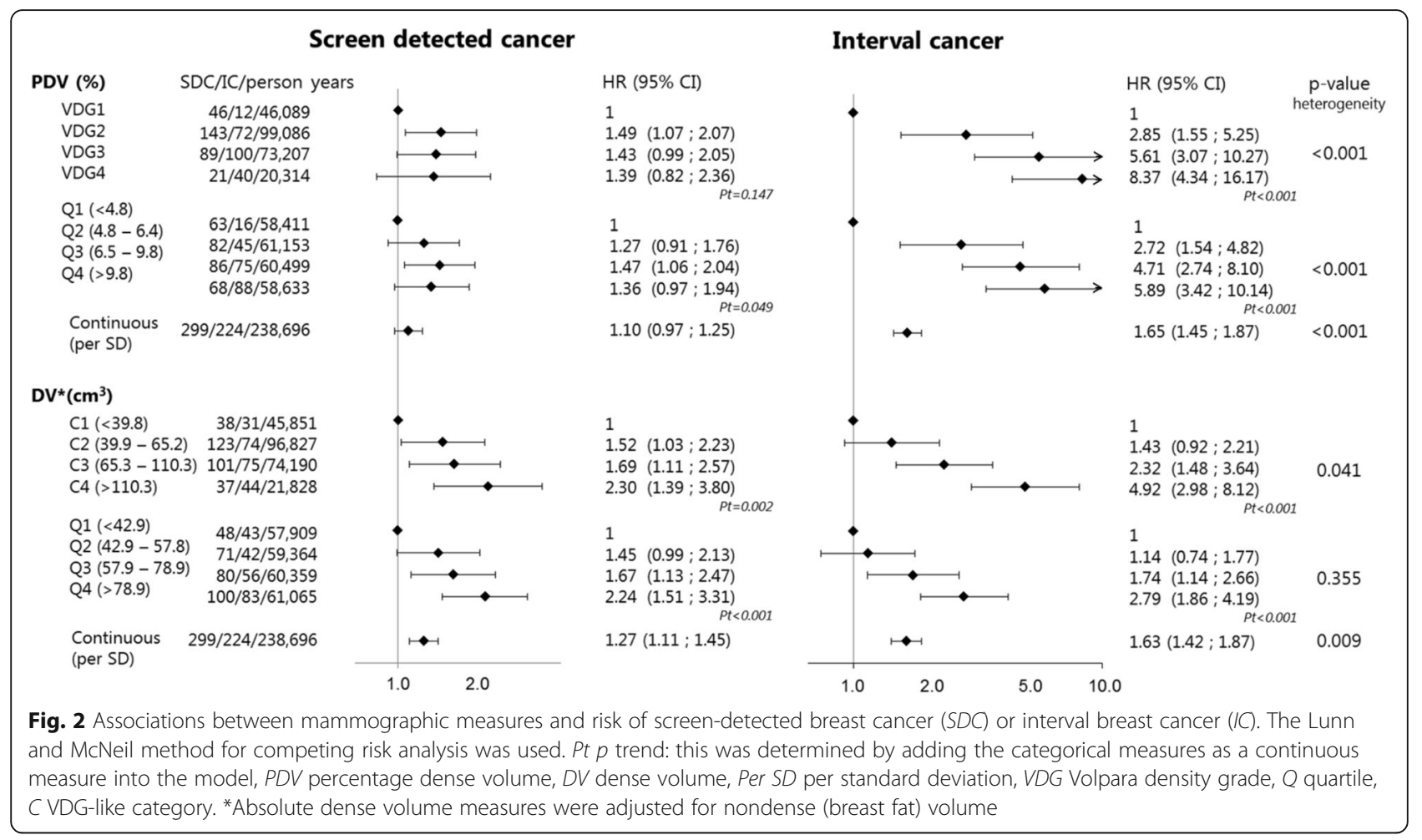

categories that are comparable to the BI-RADS categories had the highest risk estimates for association with interval breast cancers.

To our knowledge the relationship between volumetric breast density and risk of screen-detected and interval cancers, respectively, has not been studied separately before, but there are several studies on the association between volumetric breast density, determined on digital mammograms, and total breast cancer risk. An overview of their design and results is given in Additional file 3: Table S1. Four of the five presented studies examined both absolute and percentage dense volume in relation to breast cancer risk. In general, the risk estimates for percentage dense volume are somewhat higher than for absolute dense volume [15-18]. Although our risk estimates for dense volume and percentage dense volume are in line with those of the studies presented in Additional file 3: Table S1, our percentage dense volume risk estimates (VDG, PDV) seem to be slightly lower than in the other studies [15-18]. This could be explained by the fact that we do not have information on body mass index (BMI) or other risk factors in the routine screening program and therefore could not adjust for these risk factors. In particular the adjustment for BMI is known to increase the risk estimates for percent density measures. Despite this lack of adjustment for BMI, still the percent dense volume measures in our study appear to be more strongly related to the risk of interval cancer than the absolute dense volume measures.
As mentioned, no other studies have examined the ability of automated volumetric breast density assessment methods to identify women with a high risk of interval breast cancer. However, from a breast cancer screening perspective, this is important to know, since women with a high risk of interval breast cancer might benefit most from supplemental screening. Area-based breast density measures, such as BI-RADS density and the semi-automatic Cumulus thresholding method have been studied in relation to interval cancer risk. In these studies density was found to be more strongly related to the risk of an interval cancer than to the risk of a screen-detected cancer [4, 22-26, 29]. Boyd et al. (2014) and Krishnan et al. (2016) were the only two studies of both absolute dense area and percent dense area in relation to the mode of breast cancer detection. They too found that breast density was significantly more strongly related to the risk of interval breast cancers than of screen-detected breast cancers. This difference in effect seemed stronger for percentage dense area than for absolute dense area, although this was not formally tested [22, 29].

Likewise, in our study percentage dense volume seems to be a better marker for interval breast cancer risk than dense volume. When looking at total breast cancer risk, this difference between percentage dense volume and absolute dense volume seems less pronounced. An explanation for this finding could be that the amount of dense tissue is etiologically relevant for 
Table 4 Association between mammographic measures and screen-detected breast cancer risk - Lunn and McNeil

\begin{tabular}{|c|c|c|c|c|}
\hline & Cases/person years & $\mathrm{HR}_{1}(95 \% \mathrm{Cl})$ & $\mathrm{HR}_{2}(95 \% \mathrm{Cl})$ & $H R_{\text {invasive }}(95 \% \mathrm{Cl})$ \\
\hline \multicolumn{5}{|l|}{ Volpara density grades } \\
\hline VDG1 & $46 / 46,089$ & ref & NA & ref \\
\hline VDG2 & $143 / 99,086$ & $1.49(1.07 ; 2.07)$ & NA & $1.53(1.07 ; 2.21)$ \\
\hline VDG3 & $89 / 73,207$ & $1.43(0.99 ; 2.05)$ & NA & $1.41(0.94 ; 2.10)$ \\
\hline VDG4 & $21 / 20,314$ & $1.39(0.82 ; 2.36)$ & NA & $1.41(0.79 ; 2.54)$ \\
\hline$P$ trend & & 0.147 & & 0.219 \\
\hline \multicolumn{5}{|l|}{ Percent dense volume (in quartiles) } \\
\hline Q1 (<4.8\%) & $63 / 58,411$ & ref & NA & ref \\
\hline Q2 (4.8-6.4\%) & $82 / 61,153$ & $1.27(0.91 ; 1.76)$ & NA & $1.27(0.89 ; 1.81)$ \\
\hline Q3 (6.4-9.8\%) & $86 / 60,499$ & $1.47(1.06 ; 2.04)$ & NA & $1.52(1.06 ; 2.17)$ \\
\hline Q4 (>9.8\%) & $68 / 58,633$ & $1.36(0.97 ; 1.94)$ & NA & $1.27(0.86 ; 1.89)$ \\
\hline$P$ trend & & 0.049 & & 0.119 \\
\hline Percent dense volume (per SD increase) & $299 / 238,696$ & $1.10(0.97 ; 1.25)$ & NA & $1.08(0.94 ; 1.24)$ \\
\hline \multicolumn{5}{|l|}{ Dense volume (VDG-like categories) } \\
\hline $\mathrm{C} 1\left(<39.8 \mathrm{~cm}^{3}\right)$ & $38 / 45,851$ & ref & ref & ref \\
\hline C2 $\left(39.9-65.2 \mathrm{~cm}^{3}\right)$ & $123 / 96,827$ & $1.55(1.08 ; 2.23)$ & $1.52(1.03 ; 2.23)$ & $1.76(1.14 ; 2.72)$ \\
\hline C3 $\left(65.3-110.1 \mathrm{~cm}^{3}\right)$ & $101 / 74,190$ & $1.76(1.21 ; 2.56)$ & $1.69(1.11 ; 2.57)$ & $1.99(1.24 ; 3.17)$ \\
\hline $\mathrm{C} 4\left(>110.1 \mathrm{~cm}^{3}\right)$ & $37 / 21,828$ & $2.40(1.52 ; 3.79)$ & $2.30(1.39 ; 3.80)$ & $2.03(1.13 ; 3.67)$ \\
\hline$P$ trend & & $<0.001$ & 0.002 & 0.014 \\
\hline \multicolumn{5}{|l|}{ Dense volume (in quartiles)* } \\
\hline Q1 $\left(<42.9 \mathrm{~cm}^{3}\right)$ & $48 / 57,909$ & ref & ref & ref \\
\hline Q2 $\left(42.9-57.8 \mathrm{~cm}^{3}\right)$ & $71 / 59,364$ & $1.46(1.01 ; 2.10)$ & $1.45(0.99 ; 2.13)$ & $1.62(1.06 ; 2.48)$ \\
\hline Q3 $\left(57.9-78.9 \mathrm{~cm}^{3}\right)$ & $80 / 60,359$ & $1.67(1.16 ; 2.39)$ & $1.67(1.13 ; 2.47)$ & $1.86(1.21 ; 2.87)$ \\
\hline Q4 $\left(>78.9 \mathrm{~cm}^{3}\right)$ & $100 / 61,065$ & $2.22(1.57 ; 3.14)$ & $2.24(1.51 ; 3.31)$ & $2.31(1.49 ; 3.58)$ \\
\hline$P$ trend & & $<0.001$ & $<0.001$ & $<0.001$ \\
\hline Dense volume (per SD increase) & $299 / 238,696$ & $1.27(1.14 ; 1.43)$ & $1.27(1.11 ; 1.45)$ & $1.22(1.05 ; 1.41)$ \\
\hline
\end{tabular}

$H R_{1}$ Lunn and McNeil method was used for competing risk analysis, $H R_{2}$ dense volume HR's were additionally adjusted for nondense volume (quartiles), $H R_{\text {invasive }}$ Cox proportional hazards analysis with only invasive breast cancers, where age was used as the underlying time scale. Dense volume HRs were adjusted for nondense volume (quartiles). $P$ trend was determined by adding the categorical measures as a continuous measure into the model, $N A$ Not applicable "Continuous measures were natural-logarithm-transformed to establish normal distributions

breast cancer risk, and that when this dense tissue is occupying the larger part of the breast volume, these women will run a higher risk that a tumor will be masked at mammography and later diagnosed as interval cancer. If on the other hand, this same amount of dense tissue is accompanied by a large amount of fat tissue, large parts of the mammogram may still be well "readable" for cancer detection, and the probability that this tumor is detected at the screening examination will become higher.

Besides masking, another possible explanation for the relatively larger number of interval breast cancers in women with dense breasts is tumor aggressiveness. It is possible that tumors in dense breasts are more aggressive (grow faster) than tumors in nondense breasts. However, although some studies report that breast density is more strongly related to estrogen receptor (ER)- negative tumors than to ER-positive tumors, this was not confirmed in other studies [30-34].

When interpreting our results, it should be kept in mind that we excluded prevalent breast cancer cases (screen-detected cancers diagnosed as a result of the first digital screening examination). The proportion of interval cancers is therefore larger (43\%) in the current study than in the Dutch biennial screening program (26\%). As interval cancers are relatively more common in dense breasts, the association between breast density and total breast cancer risk is probably somewhat overestimated. However, this is not the case for analyses separated for screen-detected and interval breast cancers.

In most European countries women are screened from the age of 50 years. In the USA most women are screened from the age of 40 years. In addition, biennial screening is common in European countries, while in 
Table 5 Association between mammographic measures and interval breast cancer risk - Lunn and McNeil

\begin{tabular}{|c|c|c|c|c|}
\hline & Cases/person years & $\mathrm{HR}_{1}(95 \% \mathrm{Cl})$ & $\mathrm{HR}_{2}(95 \% \mathrm{Cl})$ & $H R_{\text {invasive }}(95 \% \mathrm{Cl})$ \\
\hline \multicolumn{5}{|l|}{ Volpara density grades } \\
\hline VDG1 & $12 / 46,089$ & ref & NA & ref \\
\hline VDG2 & 72/99,086 & $2.85(1.55 ; 5.25)$ & NA & $3.03(1.61 ; 5.73)$ \\
\hline VDG3 & $100 / 73,207$ & $5.61(3.07 ; 10.27)$ & NA & $6.01(3.20 ; 11.27)$ \\
\hline VDG4 & $40 / 20,314$ & $8.37(4.34 ; 16.17)$ & NA & $9.30(4.71 ; 18.37$ \\
\hline$P$ trend & & $<0.001$ & & $<0.001$ \\
\hline \multicolumn{5}{|l|}{ Percent dense volume (in quartiles) } \\
\hline Q1 (<4.8\%) & $16 / 58,411$ & ref & NA & ref \\
\hline Q2 (4.8-6.4\%) & $45 / 61,153$ & $2.72(1.54 ; 4.82)$ & NA & $2.78(1.55 ; 5.01)$ \\
\hline Q3 (6.4-9.8\%) & $75 / 60,499$ & $4.71(2.74 ; 8.10)$ & NA & $4.99(2.86 ; 8.72)$ \\
\hline Q4 (>9.8\%) & $88 / 58,633$ & $5.89(3.42 ; 10.14)$ & NA & $6.22(3.56 ; 10.89)$ \\
\hline$P$ trend & & $<0.001$ & & $<0.001$ \\
\hline Percent dense volume (per SD increase) ${ }^{\#}$ & $224 / 238,696$ & $1.65(1.45 ; 1.87)$ & NA & $1.68(1.48 ; 1.91$ \\
\hline \multicolumn{5}{|l|}{ Dense volume (VDG-like categories) } \\
\hline $\mathrm{C} 1\left(<39.8 \mathrm{~cm}^{3}\right)$ & $31 / 45,851$ & ref & ref & ref \\
\hline C2 $\left(39.9-65.2 \mathrm{~cm}^{3}\right)$ & $74 / 96,827$ & $1.13(0.75 ; 1.73)$ & $1.43(0.92 ; 2.21)$ & $1.48(0.95 ; 2.30)$ \\
\hline C3 $\left(65.3-110.1 \mathrm{~cm}^{3}\right)$ & $75 / 74,190$ & $1.51(0.99 ; 2.29)$ & $2.32(1.48 ; 3.64)$ & $2.32(1.47 ; 3.67)$ \\
\hline$C 4\left(>110.1 \mathrm{~cm}^{3}\right)$ & $44 / 21,828$ & $3.01(1.89 ; 4.79)$ & $4.92(2.98 ; 8.12)$ & $5.24(3.16 ; 8.70)$ \\
\hline$P$ trend & & $<0.001$ & $<0.001$ & $<0.001$ \\
\hline \multicolumn{5}{|l|}{ Dense volume (in quartiles)* } \\
\hline Q1 $\left(<42.9 \mathrm{~cm}^{3}\right)$ & $43 / 57,909$ & ref & ref & ref \\
\hline Q2 $\left(42.9-57.8 \mathrm{~cm}^{3}\right)$ & $42 / 59,364$ & $0.95(0.62 ; 1.46)$ & $1.14(0.74 ; 1.77)$ & $1.22(0.78 ; 1.90)$ \\
\hline Q3 $\left(57.9-78.9 \mathrm{~cm}^{3}\right)$ & $56 / 60,359$ & $1.25(0.84 ; 1.87)$ & $1.74(1.14 ; 2.66)$ & $1.83(1.19 ; 2.81)$ \\
\hline Q4 $\left(>78.9 \mathrm{~cm}^{3}\right)$ & $83 / 61,065$ & $1.83(1.26 ; 2.66)$ & $2.79(1.86 ; 4.19)$ & $2.89(1.91 ; 4.38)$ \\
\hline$P$ trend & & $<0.001$ & $<0.001$ & $<0.001$ \\
\hline Dense volume (per SD increase) ${ }^{\#}$ & $224 / 238,696$ & $1.39(1.22 ; 1.59)$ & $1.63(1.42 ; 1.87)$ & $1.65(1.44 ; 1.90)$ \\
\hline
\end{tabular}

$H R_{1}$ Lunn and McNeil method was used for competing risk analysis, $H R_{2}$ dense volume HRs were adjusted for nondense volume (quartiles), $H R_{\text {invasive }}$ Cox proportional hazards analysis with only invasive breast cancers, where age was used as the underlying time scale. Dense volume HRs were adjusted for nondense volume (quartiles). $P$ trend was determined by adding the categorical measures as a continuous measure into the model, NA Not applicable

"Continuous measures were natural-logarithm-transformed to establish normal distributions

the USA many women are screened yearly. We therefore looked at interval cancers diagnosed within the first year after a screening mammogram. We observed that $22 \%$ of the interval cancers in the group with the lowest breast density was found in that first year, while this was $60 \%$ in the group with the highest breast density, indicating an even stronger association between breast density and risk of interval breast cancer within the first year after screening.

A limitation of our study is that we could not compare the association between the automated volumetric density measurements and breast cancer risk with those of other commonly used breast density measurements such as BI-RADS. The reason for this is that BI-RADS density readings are not routinely performed in the Dutch breast cancer screening program. Previous studies directly comparing automated density measuring methods to BI-
RADS density readings and other breast density measurements in relation to breast cancer risk identified similar positive associations for all density measurements, although they may differ in their classification of which women have dense breasts and which women have not $[15-18,35,36]$. It remains as yet unknown which method most accurately reflects true density [17]. The method that we used in our study has been validated against MRI measurements of the fibroglandular tissue volume and by its fully automatic nature this gives objective and reproducible density measurements [13]. Although BI-RADS density is known to be strongly associated with breast cancer and interval breast cancer risk, the inter-reader reproducibility using this method is moderate [8-12]. A critical issue now in the discussion of the value of supplemental imaging for women with dense breasts is that it may depend on the radiologist 
who interprets the mammograms as to whether a woman is classified as having dense breasts or not [12].

Another limitation is that at the time the mammographic examinations were performed, the $\mathrm{CC}$ view was not a routine view in our screening program. The $\mathrm{CC}$ view was only taken when women had their first screening examination, or on indication, e.g. because of high breast density or suspect lesions. Although for robustness of volumetric breast density estimates it may have been preferable to calculate density on MLO and CC views, in our study we did this on the MLO view only, to prevent effect estimates from being biased by the selective availability of the $\mathrm{CC}$ views.

A final limitation is that we only had information about age as an important confounder. Therefore, adjustment of the risk estimates for other confounders was not possible. The inability to adjust for parity and other reproductive factors may have overestimated the risk estimates somewhat both for dense volume and percent dense volume. Not adjusting for BMI is expected to have led to an underestimation of the risk estimate of percentage dense volume in particular, but all in all, the risk estimates for total breast cancer risk are quite comparable to what has been published in other papers on volumetric breast density [15-18, 37]. Besides that, our study could be seen as an example for other screening programs where often little or no information is available on risk factors other than age.

Strengths of our study are the large cohort, the availability of unprocessed digital data for all its participants and its follow up since 2003 when digital mammography screening was introduced in the Netherlands. Due to linkage with the Netherlands Cancer Registry, which covers all residents of the Netherlands and is more than 95\% complete, we have virtually complete information not only on screen-detected, but also on interval breast cancers.

\section{Conclusion}

We found that automated breast density measures can be used for breast cancer risk assessment and in particular, for the risk of a tumor that is not detected at the breast cancer screening. Automated measures are especially useful in programs where BI-RADS is not routinely determined or to improve reproducibility.

\section{Additional files}

Additional file 1: Figure S1. Associations between mammographic density measures and breast cancer risk (second category used as reference). In the Cox proportional hazards analyses age was used as the underlying time scale. Pt $p$ trend: this was determined by adding the categorical measures as a continuous measure into the model, PDV percentage dense volume, DV dense volume, Per SD per standard deviation. *Absolute dense volume measures are adjusted for nondense (breast fat) volume. (DOCX $185 \mathrm{~kb}$ )
Additional file 2: Figure S2. Mammographic measures and screendetected, and interval breast cancer risk (second category used as reference). Lunn and McNeil method was used for competing risk analysis. Pt $p$ trend: this was determined by adding the categorical measures as a continuous measure into the model, PDV percentage dense volume, DV dense volume, Per SD per standard deviation, SDC screen-detected cancer, $/ C$ interval cancer. *Absolute dense volume measures are adjusted for nondense (breast fat) volume. (DOCX $890 \mathrm{~kb}$ )

Additional file 3: Table S1. Overview of associations between volumetric breast density measures (measured with Volpara) and breast cancer risk. Overview of studies that also determined the association between Volpara density grade categories and breast cancer risk. (DOCX 23 kb)

\section{Abbreviations}

BI-RADS: Breast Imaging - Reporting and Data System; BMI: Body mass index; CC: Craniocaudal; DM: Digital mammography; DV: Dense volume; ER: Estrogen receptor; HR: Hazard ratio; IQR: Interquartile range; Ln: Natural logarithm; MLO: Mediolateral oblique; MRI: Magnetic resonance imaging; NDV: Nondense (breast fat) volume; PDV: Percentage dense volume;

Q: Quartile; SD: Standard deviation; VDGs: Volpara density grades

\section{Acknowledgements}

We want to thank the Foundation of Population Screening Mid-West (The Netherlands) for providing data.

\section{Funding}

The research leading to these results has received funding from the European Union's Seventh Framework Programme FP7 under grant agreement no 306088 and a personal grant of the Dutch Cancer Society (to CG) (grant number KWF UU 2009-4348). The funding bodies were not involved in the design of the study or the collection, analysis and interpretation of data or in writing the manuscript.

\section{Availability of data and materials}

The data that support the findings of this study are available from the Foundation of Population Screening Mid-West (Utrecht, The Netherlands) but restrictions apply to the availability of these data, which were used under license for the current study, and so are not publicly available. Data are however available from the authors upon reasonable request and with permission of the Foundation of Population Screening Mid-West (Utrecht, The Netherlands)

\section{Authors' contributions}

JW performed the data analysis and wrote the manuscript. KH and NK collected the data used in this study; in addition they critically reviewed the manuscript. WV and RM were involved in the conception and design of the study and critically reviewed the manuscript. CVG and PP were the project leaders of this research project and were therefore involved in all stages of this study. All authors read and approved the final manuscript and agree to be accountable for all aspects of the work.

\section{Competing interests}

NK reports to be one of the co-founders of Volpara Solutions, which develops and markets the breast density measurement software Volpara used in this study. In addition, NK has a patent pending and is co-founder of two other companies in the field of breast imaging next to his position as professor in the University. The two companies are Qview Medical (Los, Altos, CA, USA), and ScreenPoint Medical (Nijmegen, the Netherlands). These companies develop products for computer aided detection of breast cancer, in whole breast ultrasound and in mammography respectively. CVG reports a grant from Bayer Healthcare, and non-financial support from Volpara Solutions outside the submitted work. In addition, RM reports grants, personal fees, and non-financial support from Siemens Healthcare and grants and personal fees from Bayer Healthcare outside the submitted work. RM also reports a research contract with Seno Medical and he reports to be a scientific advisor for ScreenPoint Medical (Nijmegen, the Netherlands) outside the submitted work. 


\section{Ethics approval and consent to participate}

By participating in the Dutch screening program, women consent to their data being used for evaluation and improvement of the screening, unless they have indicated otherwise. The research ethics committee of the Radboud University Nijmegen Medical Centre declared that this study does not fall within the remit of the Medical Research Involving Human Subjects Act. Therefore, this study could be carried out (in the Netherlands) without approval by an accredited research ethics committee.

\section{Publisher's Note}

Springer Nature remains neutral with regard to jurisdictional claims in published maps and institutional affiliations.

\section{Author details}

'Julius Center for Health Sciences and Primary Care, University Medical Center Utrecht, P.O. Box 855003508 GA Utrecht, The Netherlands. ${ }^{2}$ Department of Radiology and Nuclear Medicine, Radboud University Medical Center, Geert Grooteplein 10, 6525 GA Nijmegen, The Netherlands. ${ }^{3}$ MRC-PHE Centre for Environment and Health, Department of Epidemiology and Biostatistics, School of Public Health, Imperial College London, St. Mary's Campus, Norfolk Place, W2 1PG London, UK. ${ }^{4}$ Department of Radiology, University Medical Center Utrecht, P.O. Box 855003508 GA Utrecht, The Netherlands.

Received: 9 December 2016 Accepted: 19 May 2017

Published online: 05 June 2017

\section{References}

1. Vachon CM, van Gils CH, Sellers TA, Ghosh K, Pruthi S, Brandt KR, Pankratz VS. Mammographic density, breast cancer risk and risk prediction. Breast Cancer Res. 2007;9(6):217.

2. McCormack VA, Dos Santos Silva I. Breast density and parenchymal patterns as markers of breast cancer risk: a meta-analysis. Cancer Epidemiol Biomark Prev. 2006;15(6):1159-69.

3. Pettersson A, Graff RE, Ursin G, Santos Silva ID, McCormack V, Baglietto L, Vachon C, Bakker MF, Giles GG, Chia KS et al. Mammographic density phenotypes and risk of breast cancer: a meta-analysis. J Natl Cancer Inst. 2014; 106(5).

4. Boyd NF, Guo H, Martin LJ, Sun L, Stone J, Fishell E, Jong RA, Hislop G, Chiarelli A, Minkin S, et al. Mammographic density and the risk and detection of breast cancer. N Engl J Med. 2007;356(3):227-36.

5. Kerlikowske K. The mammogram that cried Wolfe. New Engl J Med. 2007; 356(3):297-300.

6. Wanders JO, Holland K, Veldhuis WB, Mann RM, Pijnappel RM, Peeters PH, van Gils CH, Karssemeijer N. Volumetric breast density affects performance of digital screening mammography. Breast Cancer Res Treat. 2017;162(1):95-103.

7. D'Orsi CJ, Sickles EA, Mendelson EB EAM. ACR BI-RADS ${ }^{\circledR}$ Atlas, Breast Imaging Reporting and Data System. Reston, VA: American College of Radiology; 2013

8. Ciatto S, Houssami N, Apruzzese A, Bassetti E, Brancato B, Carozzi F, Catarzi S, Lamberini MP, Marcelli G, Pellizzoni R, et al. Categorizing breast mammographic density: intra- and interobserver reproducibility of BI-RADS density categories. Breast. 2005;14(4):269-75.

9. Gard CC, Aiello Bowles EJ, Miglioretti DL, Taplin SH, Rutter CM. Misclassification of Breast Imaging Reporting and Data System (BI-RADS) Mammographic Density and Implications for Breast Density Reporting Legislation. Breast J. 2015;21(5):481-9.

10. Gweon HM, Youk JH, Kim JA, Son EJ. Radiologist assessment of breast density by BI-RADS categories versus fully automated volumetric assessment. AJR Am J Roentgenol. 2013;201(3):692-7.

11. Redondo A, Comas M, Macia F, Ferrer F, Murta-Nascimento C, Maristany MT, Molins E, Sala M, Castells X. Inter- and intraradiologist variability in the BlRADS assessment and breast density categories for screening mammograms. Br J Radiol. 2012;85(1019):1465-70.

12. Sprague BL, Conant EF, Onega T, Garcia MP, Beaber EF, Herschorn SD, Lehman $C D$, Tosteson AN, Lacson R, Schnall MD, et al. Variation in mammographic breast density assessments among radiologists in clinical practice: a multicenter observational study. Ann Intern Med. 2016;165(7):457-64.

13. Gubern-Merida A, Kallenberg M, Platel B, Mann RM, Marti R, Karssemeijer N, et al. Volumetric breast density estimation from full-field digital mammograms: a validation study. PLoS One. 2014;9(1):e85952.
14. Highnam R, Brady M, Yaffe MJ, Karssemeijer N, Harvey J. Robust breast composition measurement - Volpara (TM). Lect Notes Comput Sci. 2010; 6136:342-9

15. Eng A, Gallant Z, Shepherd J, McCormack V, Li J, Dowsett M, Vinnicombe S, Allen S, dos-Santos-Silva I. Digital mammographic density and breast cancer risk: a case-control study of six alternative density assessment methods. Breast Cancer Res. 2014;16(5):439.

16. Brand JS, Czene K, Shepherd JA, Leifland K, Heddson B, Sundbom A, Eriksson M, Li J, Humphreys K, Hall P. Automated measurement of volumetric mammographic density: a tool for widespread breast cancer risk assessment. Cancer Epidemiol Biomark Prev. 2014;23(9):1764-72.

17. Brandt KR, Scott CG, Ma L, Mahmoudzadeh AP, Jensen MR, Whaley DH, Wu FF, Malkov S, Hruska CB, Norman AD et al. Comparison of clinical and automated breast density measurements: implications for risk prediction and supplemental screening. Radiology. 2015;279(3):710-9

18. Jeffers AM, Sieh W, Lipson JA, Rothstein JH, McGuire V, Whittemore AS, Rubin DL. Breast Cancer Risk and Mammographic Density Assessed with Semiautomated and Fully Automated Methods and BI-RADS. Radiology. 2016:282(2):348-55.

19. Ursin G, Hovanessian-Larsen L, Parisky YR, Pike MC, Wu AH. Greatly increased occurrence of breast cancers in areas of mammographically dense tissue. Breast Cancer Res. 2005;7(5):R605-8.

20. Haars G, van Noord PA, van Gils CH, Grobbee DE, Peeters PH. Measurements of breast density: no ratio for a ratio. Cancer Epidemiol Biomark Prev. 2005; 14(11 Pt 1):2634-40.

21. Kerlikowske K, Zhu W, Tosteson AN, Sprague BL, Tice JA, Lehman CD, Miglioretti DL. Identifying women with dense breasts at high risk for interval cancer: a cohort study. Ann Intern Med. 2015;162(10):673-81.

22. Boyd NF, Huszti E, Melnichouk O, Martin LJ, Hislop G, Chiarelli A, Yaffe MJ, Minkin S. Mammographic features associated with interval breast cancers in screening programs. Breast Cancer Res. 2014;16(4):417.

23. Mandelson MT, Oestreicher N, Porter PL, White D, Finder CA, Taplin SH, White E. Breast density as a predictor of mammographic detection: comparison of interval- and screen-detected cancers. J Natl Cancer Inst. 2000;92(13):1081-7.

24. Holm J, Humphreys K, Li J, Ploner A, Cheddad A, Eriksson M, Tornberg S, Hall P, Czene K. Risk factors and tumor characteristics of interval cancers by mammographic density. J Clin Oncol. 2015;33(9):1030-7.

25. Crane CE, Luke CG, Rogers JM, Playford PE, Roder DM. An analysis of factors associated with interval as opposed to screen-detected breast cancers, including hormone therapy and mammographic density. Breast. 2002;11(2):131-6.

26. Domingo L, Salas D, Zubizarreta R, Bare M, Sarriugarte G, Barata T, Ibanez J, Blanch J, Puig-Vives M, Fernandez A, et al. Tumor phenotype and breast density in distinct categories of interval cancer: results of population-based mammography screening in Spain. Breast Cancer Res. 2014;16(1):R3.

27. Lunn M, McNeil D. Applying Cox regression to competing risks. Biometrics. 1995:51(2):524-32.

28. Kleinbaum DG, Klein M. Survival analysis - a self-learning text. Third edn: Springer; 2012. pp. 455-61.

29. Krishnan K, Baglietto L, Apicella C, Stone J, Southey MC, English DR, Giles GG, Hopper JL. Mammographic density and risk of breast cancer by mode of detection and tumor size: a case-control study. Breast Cancer Res. 2016; 18(1):63.

30. Bertrand KA, Tamimi RM, Scott CG, Jensen MR, Pankratz V, Visscher D, Norman A, Couch F, Shepherd J, Fan B, et al. Mammographic density and risk of breast cancer by age and tumor characteristics. Breast Cancer Res. 2013;15(6):R104

31. Pollan M, Ascunce N, Ederra M, Murillo A, Erdozain N, Ales-Martinez J, Pastor-Barriuso R. Mammographic density and risk of breast cancer according to tumor characteristics and mode of detection: a Spanish population-based case-control study. Breast Cancer Res. 2013;15(1):R9.

32. Razzaghi H, Troester MA, Gierach GL, Olshan AF, Yankaskas BC, Millikan RC. Association between mammographic density and basal-like and luminal A breast cancer subtypes. Breast Cancer Res. 2013;15:5. R76.

33. Yaghjyan L, Colditz GA, Collins LC, Schnitt SJ, Rosner B, Vachon C, Tamimi RM. Mammographic breast density and subsequent risk of breast cancer in postmenopausal women according to tumor characteristics. J Natl Cancer Inst. 2011;103(15):1179-89.

34. Yaghjyan L, Pettersson A, Colditz GA, Collins LC, Schnitt SJ, Beck AH, Rosner B, Vachon C, Tamimi RM. Postmenopausal mammographic breast density and subsequent breast cancer risk according to selected tissue markers. $\mathrm{Br} \mathrm{J}$ Cancer. 2015;113(7):1104-13. 
35. Seo JM, Ko ES, Han BK, Ko EY, Shin JH, Hahn SY. Automated volumetric breast density estimation: a comparison with visual assessment. Clin Radiol. 2013;68(7):690-5

36. Lee HN, Sohn YM, Han KH. Comparison of mammographic density estimation by Volpara software with radiologists' visual assessment: analysis of clinical-radiologic factors affecting discrepancy between them. Acta Radiol. 2015;56(9):1061-8.

37. Park IH, Ko K, Joo J, Park B, Jung SY, Lee S, Kwon Y, Kang HS, Lee ES, Lee KS et al. High volumetric breast density predicts risk for breast cancer in postmenopausal, but not premenopausal, Korean Women. Ann Surg Oncol. 2014:21(13):4124-32

Submit your next manuscript to BioMed Central and we will help you at every step:

- We accept pre-submission inquiries

- Our selector tool helps you to find the most relevant journal

- We provide round the clock customer support

- Convenient online submission

- Thorough peer review

- Inclusion in PubMed and all major indexing services

- Maximum visibility for your research

Submit your manuscript at www.biomedcentral.com/submit 